\title{
Division of a single free flap in multiple digit reconstruction
}

\author{
Jin Soo Kim, Cheon Ho Song, Si Young Roh, Sung Hoon Koh, Dong Chul Lee, Kyung Jin Lee \\ Department of Plastic and Reconstructive Surgery, Gwangmyeong Sungae General Hospital, Gwangmyeong, Korea
}

\begin{abstract}
Background Single free flaps are a commonly used reconstructive method for multiple soft tissue defects in digits. We analyzed the flap size, division timing, and degree of necrosis in cases with various types of flap division.

Methods We conducted a retrospective review of the medical charts of patients who had undergone single free flap reconstruction for multiple soft tissue defects across their digits from 2011 to 2020. The flap types included were the lateral arm free flap, venous forearm free flap, thenar free flap, hypothenar free flap, anterolateral thigh free flap, medial plantar free flap, and second toe pulp free flap. Flap size, anastomosed vessels, division timing, and occurrence of flap necrosis were retrospectively investigated and then analyzed using the ttest.

Results In total, 75 patients were included in the analysis. The success rate of the free flaps was $97.3 \%$. All flaps were successfully divided after at least 17 days, with a mean of 47.17 days (range, 17-243 days) for large flaps and 42.81 days (range, 20-130 days) for the medium and small flaps $(P=0.596)$. The mean area of flap necrosis was $2.38 \%$ in the large flaps and $2.58 \%$ in the medium and small flaps $(P=0.935)$. Severe necrosis of the divided flap developed in two patients who had undergone flap division at week 6 and week 34 .

Conclusions In cases where blood flow to the flap has been stable for more than 3 weeks, flap division can be safely attempted regardless of the flap size.
\end{abstract}

Keywords Free tissue flap / Soft tissue injury / Finger injury / Reconstructive surgery

Received: July 29, $2021 \bullet$ Revised: October 5, $2021 \bullet$ Accepted: October 22, 2021

pISSN: 2234-6163 • elSSN: 2234-6171 • https://doi.org/10.5999/aps.2021.01466 • Arch Plast Surg 2022;49:61-69

\author{
Correspondence: Si Young Roh \\ Department of Plastic and \\ Reconstructive Surgery, \\ Gwangmyeong Sungae General \\ Hospital, 36 Digital-ro, Gwangmyeong \\ 14241, Korea \\ Tel: +82-2-2680-7637 \\ Fax: +82-2-2615-7218 \\ E-mail: psczero@gmail.com
}

\section{INTRODUCTION}

Patients who present with degloving injuries to the hands or amputation of two or more digits are rare. Replantation is ideal but often impossible or unsuccessful. Without proper vascular tissue transplantation, necrosis of the amputated or denuded area is imminent [1-4]. The advent of microsurgery has led to the introduction of fascial free flaps, innervated fasciocutaneous flaps, and arterialized venous flaps for reconstruction [5-8]. These options are ideal for the reconstruction of soft tissue de- fects in the hand according to the size, shape, and site of each defect. Single free flaps are a reconstructive method commonly used in our institution to address the challenges encountered in degloved or amputated stumps of multiple digits. Various free flap types have been performed using various donor regions, from the upper extremities to the lower extremities. However, this procedure requires secondary division and setting.

Despite evidence that sufficient neovascularization to sustain the flap develops before 3 weeks, reconstructive surgeons have been advised to wait 3 weeks before attempting flap division [9]. 
Although we also planned flap division after 3 weeks, there were cases in which flap division was performed later due to concerns about necrosis. The timing of flap division after 3 weeks is common practice, but we had questions about whether this should be applied equally to various types of flaps. Division at 3 weeks is more difficult for large and thick flaps than for small flaps. The aim of this study was to analyze flap divisions of various types and to determine the relationship between the size of the flap, the timing of separation surgery, and the degree of necrosis.

\section{METHODS}

\section{Patients}

This was a retrospective review of patients who underwent simultaneous free flap reconstruction of multiple soft tissue defects in the hand between 2011 and 2020 at our institution. The need for patient consent was waived due to the retrospective nature of the study, and the study design was approved by the institutional review board (IRB No. KIRB-2021-N-002).

Cases of single free flaps performed across multiple digits for soft tissue reconstruction between 2011 and 2020 were investigated, including both primary and secondary operations. Various types of flaps from the upper extremity to the lower extremity were performed based on the location and the number of digits with tissue defects. The flap option was chosen after sufficient discussion with the patient about donor site mobility and cosmetics.

\section{Flap types and operative techniques \\ Lateral arm free flap (fasciocutaneous or fascial type)}

This flap contains the posterior radial collateral artery and is elevated with one dominant perforator artery and one or two venae comitantes on the lateral aspect of the upper arm. This flap may include the posterior brachial cutaneous nerve for sensation and is anastomosed to the digital artery, nerve, and subcutaneous veins.

\section{Venous forearm free flap}

This flap is harvested from the anterior portion of the ipsilateral forearm, which contains three to four veins. The flap was elevated with one afferent vein and several efferent veins. The afferent vein of the flap was anastomosed to the digital artery and the draining veins were anastomosed to the dorsal veins. The flap is arterialized with arterial inflow and venous outflow (A-V-V type).

\section{Thenar free flap}

This flap is designed from the thenar crease to the wrist crease, centering on the scaphoid tubercle. The flap is elevated with the superficial palmar branch of the radial artery to the skin and is elevated with one perforator and one or two venae comitantes. The perforator artery is anastomosed to the digital artery and the draining vein is sutured to the subcutaneous vein.

\section{Hypothenar free flap}

This flap is designed from the hypothenar eminence to ulnar and dorsal skin. The flap is elevated with one perforator artery that arises from the fourth common palmar digital artery and one subcutaneous vein, and is anastomosed to the digital artery and recipient subcutaneous vein. If complete coverage of the defect by the flap is unfeasible, a skin graft near the flap donor site at the hypothenar eminence is harvested [10].

\section{Anterolateral thigh free flap (fasciocutaneous or fascial type)}

This flap contains the descending branch of the lateral femoral circumflex artery and is elevated with one dominant perforator artery and one or two venae comitantes. In addition, the lateral femoral cutaneous nerve is included in the flap. The flap is anastomosed to the digital artery, nerve, and subcutaneous veins.

\section{Medial plantar free flap}

This flap is designed on the medial aspect of the ipsilateral plantar arch flap. It is harvested with the medial plantar perforator artery and nerve and is anastomosed to the digital artery and nerve. Several subcutaneous veins of the flap are sutured to the subcutaneous veins of the digits.

\section{Second partial toe pulp free flap}

This flap is designed on the medial side of the ipsilateral second toe pulp area. The plantar digital artery and paired nerve are elevated with several venae comitantes. The flap is anastomosed to the recipient digital artery and nerve of the injured digit. The draining veins of the flap are sutured to the subcutaneous veins of the digit.

\section{Division of the flap}

After free flap surgery, the circulation of the flap was closely monitored by measuring the capillary refill time and flap surface temperature, and the patency of the anastomosed artery was checked using a hand-held Doppler. The color change and necrosis of the flap margins were evaluated. As anticoagulant therapy, 12,000 U of heparin was administered along with $10 \mu \mathrm{g}$ of prostaglandin E1 for 1 week. In cases of syndactyly due to the flap, active and passive joint exercises of the metacarpophalangeal or proximal interphalangeal joint were started at the first postoperative week.

Flap division was planned after at least 3 weeks, which is the 
standard time used by many surgeons to safely allow neovascularization [9]. During flap monitoring, a capillary refill test was performed and changes in the flap end were measured to determine the timing of flap division surgery. For some small and thin flaps, the timing of flap division was decided by using clamping to check the blood circulation of the flap. Immediately after division of the flap during surgery, the capillary refill time of the divided flap was checked. If additional skin coverage was required during flap division surgery, such as in the web space or joint space, then a skin graft or local flap was performed using Zplasty to prevent contracture. After surgery, the color change and necrosis of the flap margins were evaluated. In some cases that involved three or more digits, flap division was performed in two steps and the second step flap division operation was performed at least 2 weeks after the first division operation.

The patients' demographics, flap type, diameter of the flap, number of anastomosed vessels, frequency of complications (e.g., infection, hematoma, and emergent re-exploration), timing of flap division surgery, and degree of flap necrosis were retrospectively investigated by evaluating their medical records and photographs. The continuous variables between the two groups in this study (the large flap group and the medium or small flap group) were compared using the $t$-test. In the interpretation of results, $\mathrm{P}$-values $<0.05$ were considered to indicate statistical significance. All statistical analyses were performed using SPSS version 28.0 (IBM Corp., Armonk, NY, USA).

\section{RESULTS}

In total, 75 patients were included in the analysis (Table 1). The success rate was approximately $97 \%$ (73/75), and only two cases of flap failure occurred. These two patients underwent a secondary groin flap and skin graft. The incidence of partial flap necrosis was $15 \%(11 / 75)$ and did not involve more than $30 \%$ of the total area in any patient. Re-exploration was performed in 7\% of patients (5/75). The mean age was 42.9 years (range, 19-64 years), and 68 patients were men and seven patients were women. Industrial accidents accounted for $91 \%$ of cases $(68 / 75)$. In total, $4 \%$ of cases $(3 / 75)$ were from a degloving injury due to a traffic accident, $4 \%$ of cases $(3 / 75)$ were from necrosis due to thermal burns, and one case of skin cancer was included. Primary reconstruction was performed in $61.3 \%$ of cases (46/75), and secondary reconstruction due to necrosis after replantation of amputated fingers or composite grafting of degloving soft tissue accounted for $28.7 \%$ of cases.

The injury location was classified as the amputation stump, dorsum of the digit, and volar aspect of the digit (Table 2). There were 35 cases of resurfacing two digits and 38 cases of

Table 1. Incidence of complications by flap type

\begin{tabular}{|c|c|c|c|c|}
\hline Type of free flap & No. & Partial loss, №. (\%) & Flap failure, №. (\%) & Re-exploration, №. (\%) \\
\hline Lateral arm free flap (fasciocutaneous type) & 4 & 0 & 0 & 0 \\
\hline Lateral arm free flap (fascial type) & 4 & 0 & 0 & 0 \\
\hline Venous forearm free flap & 2 & 0 & 0 & 0 \\
\hline Thenar free flap & 15 & $1(7)$ & 0 & $1(7)$ \\
\hline Hypothenar free flap & 3 & $1(33)$ & 0 & 0 \\
\hline Anterolateral thigh free flap (fasciocutaneous type) & 37 & $7(19)$ & $2(5)$ & $4(11)$ \\
\hline Anterolateral thigh free flap (fascial type) & 7 & $1(14)$ & 0 & 0 \\
\hline Medial plantar free flap & 1 & $1(100)$ & 0 & 0 \\
\hline Partial second toe pulp free flap & 1 & 0 & 0 & 0 \\
\hline Total & 75 & $11(15)$ & $2(3)$ & $5(7)$ \\
\hline
\end{tabular}

Table 2. Indication of flap types according to recipient site

\begin{tabular}{|c|c|c|c|c|c|c|c|}
\hline \multirow[b]{2}{*}{ Recipient sites } & \multicolumn{7}{|c|}{ Flap types (№.) } \\
\hline & $\begin{array}{l}\text { Anterolateral } \\
\text { thigh free flap }{ }^{a)}\end{array}$ & $\begin{array}{l}\text { Lateral arm } \\
\text { free flap }{ }^{b)}\end{array}$ & $\begin{array}{l}\text { Venous forearm } \\
\text { free flap }\end{array}$ & $\begin{array}{l}\text { Thenar free } \\
\text { flap }\end{array}$ & $\begin{array}{l}\text { Medial plantar } \\
\text { free flap }\end{array}$ & $\begin{array}{l}\text { Hypo-thenar } \\
\text { free flap }\end{array}$ & $\begin{array}{l}\text { Partial second } \\
\text { toe pulp free flap }\end{array}$ \\
\hline \multicolumn{8}{|l|}{ Two digits $(n=35)$} \\
\hline Amputation stumps & $\mathrm{Y}(6)$ & $Y(4)$ & $\mathrm{N}$ & $Y(10)$ & $\mathrm{N}$ & $\mathrm{N}$ & $\mathrm{N}$ \\
\hline Dorsal side & $Y(3)$ & $Y(1)$ & $\mathrm{Y}(2)$ & $Y(1)$ & $\mathrm{N}$ & $Y(2)$ & $\mathrm{N}$ \\
\hline Volar side & $\mathrm{Y}(3)$ & $\mathrm{N}$ & $\mathrm{N}$ & $\mathrm{Y}(2)$ & $\mathrm{N}$ & N & $Y(1)$ \\
\hline \multicolumn{8}{|c|}{ Three or more digits $(n=38)$} \\
\hline Amputation stumps & $Y(25)$ & $Y(3)$ & N & Y (3) & $Y(1)$ & $\mathrm{N}$ & $\mathrm{N}$ \\
\hline Dorsal side & $Y(4)$ & $\mathrm{N}$ & $\mathrm{N}$ & $\mathrm{N}$ & $\mathrm{N}$ & $Y(1)$ & $\mathrm{N}$ \\
\hline Volar side & $Y(1)$ & $\mathrm{N}$ & $\mathrm{N}$ & $\mathrm{N}$ & $\mathrm{N}$ & $\mathrm{N}$ & $\mathrm{N}$ \\
\hline
\end{tabular}

$\mathrm{Y}$, indicates that a flap was used; $\mathrm{N}$, indicates that a flap was not used.

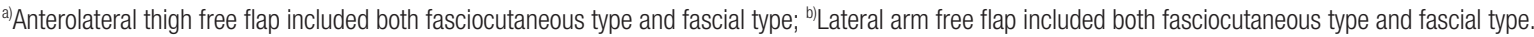


soft tissue defects in three or more digits. An anterolateral thigh free flap was used in 12 cases of resurfacing two digits and in 30 cases of injury to three or more digits (Fig. 1). In three patients, this flap was used for all five amputation stumps. A lateral arm free flap was used in five cases of resurfacing two amputation stumps and the dorsal sides of two digits and in three cases of injury with three amputation stumps. A venous forearm free flap was used in two cases of a degloving injury to the dorsal side of two digits. Thenar free flaps were used in 10 cases of resurfacing two amputation stumps and in three cases of injury with three amputation stumps. Three cases involved resurfacing the volar and dorsal sides of two digits. A hypothenar free flap was used in two degloving injury cases for the dorsal side of two digits and, in one case, three digits. A medial plantar free flap and second partial toe pulp free flap were used in one case of injury to the amputation stumps of three digits and in one case of injury to the pulp of two digits.

Based on the flap size, patients were classified into two groups: a large flap group and a medium and small flap group (Table 3 ). The anterolateral thigh free flap (fasciocutaneous or fascial
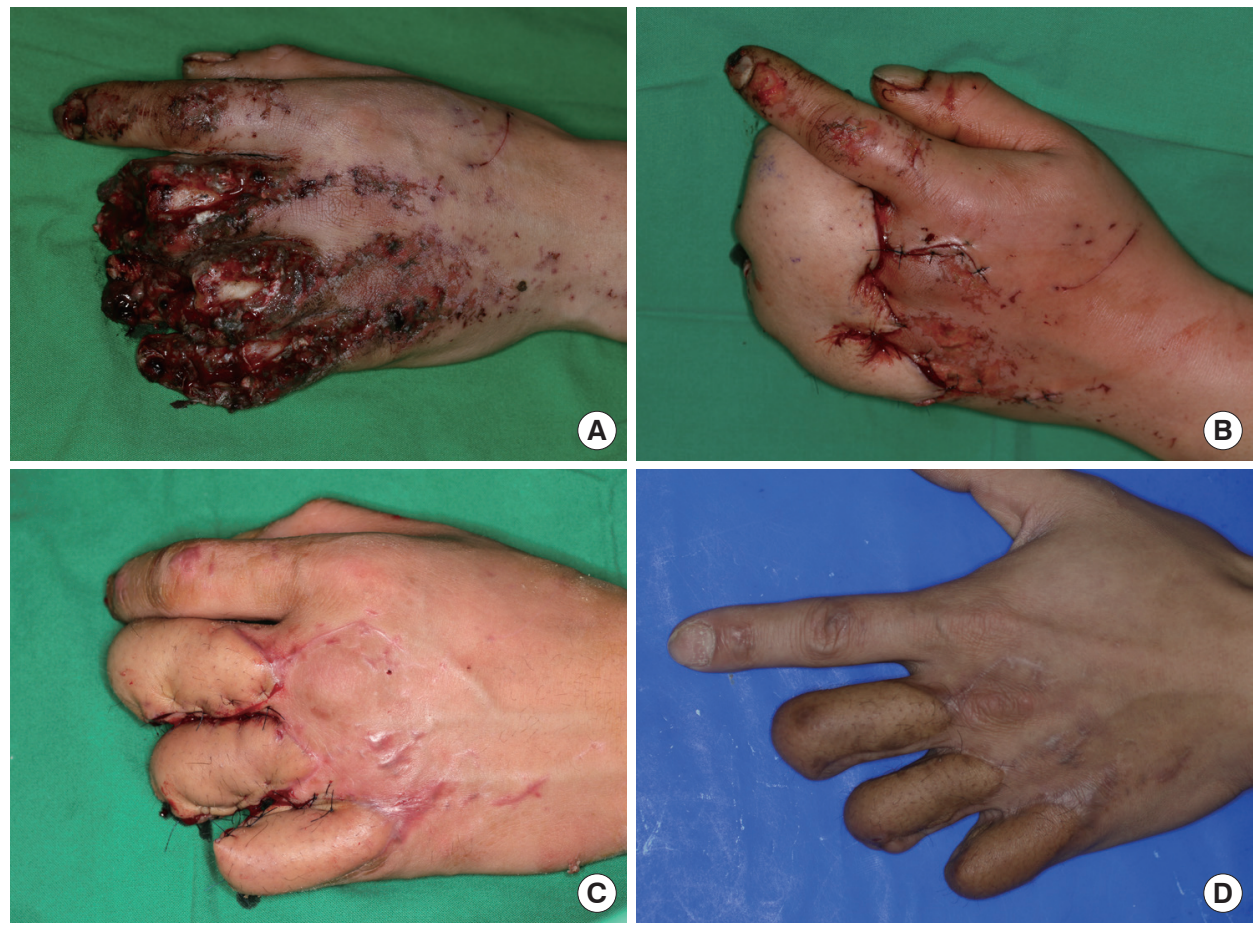

Fig. 1. Case of anterolateral thigh free flap. (A) A 27-year-old male patient has injured in a car accident with degloving injuries of adjacent three digits. After debridement of contaminated tissue, the digits were amputated at the proximal interphalangeal joint. (B) A $15 \times 7 \mathrm{~cm}$ fasciocutaneous flap, which contained one perforator artery, two venae comitantes, and one cutaneous nerve, was harvested. Each artery and nerve were anastomosed to the ulnar digital arteries and nerve of the long finger. The draining veins were sutured to the dorsal vein of the long finger and volar vein of the small fingers. (C) A single flap division was performed 24 days after the first surgery. (D) At the 15-month follow-up, the divided flaps survived completely without the need for additional surgery.

Table 3. Classification of flap types according to size

\begin{tabular}{|c|c|c|c|}
\hline Flap type & No. of cases & Mean diameter (cm) & Range (cm) \\
\hline \multicolumn{4}{|l|}{ Large } \\
\hline Anterolateral thigh (fasciocutaneous) & 35 & $14.6 \times 6.5$ & $8 \times 4-20 \times 10$ \\
\hline Anteriolateral thigh (fascial) & 7 & $11.6 \times 4.4$ & $7 \times 4-18 \times 5$ \\
\hline \multicolumn{4}{|l|}{ Medium and small } \\
\hline Lateral arm (fasciocutaneous) & 4 & $7.5 \times 3.3$ & $7 \times 2.5-8 \times 4$ \\
\hline Lateral arm (fascial) & 4 & $6.3 \times 3.3$ & $4 \times 3-10 \times 4$ \\
\hline Venous forearm & 2 & $6.5 \times 3.0$ & $5 \times 3-8 \times 3$ \\
\hline Thenar & 15 & $6.2 \times 2.2$ & $5 \times 1.5-11 \times 3$ \\
\hline Medial plantar & 2 & $6.5 \times 3.0$ & $6 \times 3-7 \times 3$ \\
\hline Hypothenar & 3 & $3.5 \times 2.0$ & $2.5 \times 1-4 \times 3$ \\
\hline Second toe pulp & 1 & $3.0 \times 1.0$ & $3.0 \times 1.0$ \\
\hline
\end{tabular}


type) was considered a large flap with a long axis mean diameter of $>10 \mathrm{~cm}$. Flaps with a mean diameter of $>6 \mathrm{~cm}$ were considered medium-sized flaps, including lateral arm free flaps, venous forearm free flaps, thenar free flaps, and medial plantar free flaps. Flaps with a mean diameter of $<6 \mathrm{~cm}$ were considered small flaps, including hypothenar free flaps and second toe pulp free flaps. The mean numbers of anastomosed arteries and veins in the large flap group were 1.09 and $1.81(\mathrm{P}=0.265)$, respectively, and 1.03 and $1.55(\mathrm{P}=0.166)$ in the medium and small flap group, respectively (Table 4).

In total, 73 patients underwent flap division surgery, which was performed at an average of 47.17 days after initial flap surgery (range, 17-243 days) in the large flap group and 42.81 days (range, 20-130 days) in the medium and small flap group (Fig. 2 ). This difference between the groups was not statistically significant $(\mathrm{P}=0.596)$ (Table 5). The divided flaps had partial necrosis in $9.6 \%$ of cases $(7 / 73)$, but most cases were successfully divided after at least 17 days. The mean area of flap necrosis was $2.38 \%$ in the large flap group and $2.58 \%$ in the medium and small flap group $(\mathrm{P}=0.935)$ (Table 6). Severe necrosis with an area of more than $50 \%$ of the divided flap developed in one case of large flap division at 34 weeks and in one case of medium flap division at 6 weeks.

\section{DISCUSSION}

In cases of degloving injuries to the hand, ischemia and necrosis of the denuded area may be imminent without proper vascular tissue transplantation [1-4]. Importantly, the soft tissue envelope is necessary for blood supply to the distal region. In cases involving amputation of two or more digits, replantation is ideal but often impossible or unsuccessful. The reconstruction goals, in situations where simultaneous coverage of multiple soft tissue defects in the hand is required, are preservation of length, im-

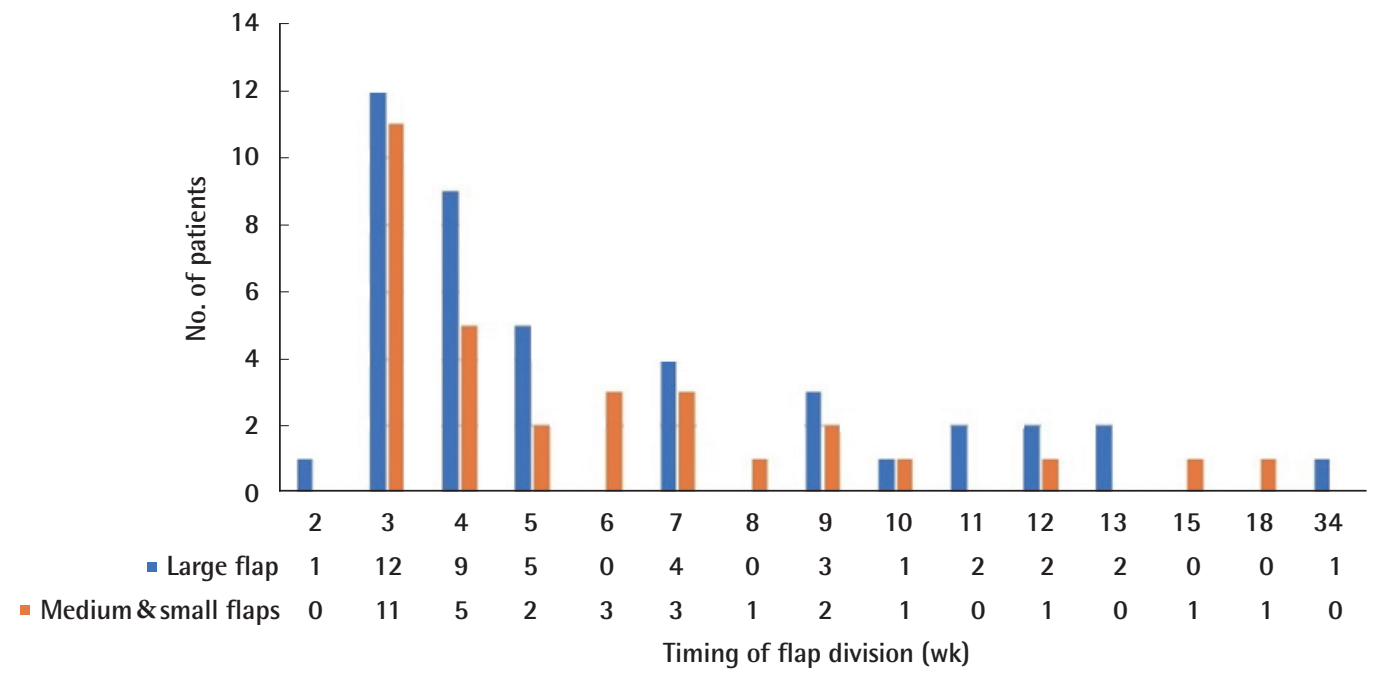

Fig. 2. Comparison of the timing of flap division between the large and small or medium flap groups.

Table 4. Comparison of flap type and the number of anastomosed vessels

\begin{tabular}{lcc}
\hline \multirow{2}{*}{ Flap size } & \multicolumn{2}{c}{ No. of anastomosed vessels, mean \pm SD } \\
\cline { 2 - 3 } & Artery $^{\text {a) }}$ & Vein $^{\text {b) }}$ \\
\hline Large flap $(n=42)$ & $1.09 \pm 0.30$ & $1.81 \pm 0.80$ \\
Medium \& small flap $(n=31)$ & $1.03 \pm 1.80$ & $1.55 \pm 0.77$ \\
\hline
\end{tabular}

a) $\mathrm{P}=0.265$ and ${ }^{\mathrm{b} P} \mathrm{P}=0.166$ as calculated using the t-test.
Table 5. Comparison of flap type and division timing

\begin{tabular}{lcc}
\hline \multirow{2}{*}{ Flap size } & \multicolumn{2}{c}{ Days after free flap } \\
\cline { 2 - 3 } & Mean $\left.\pm \mathrm{SD}^{\mathrm{a}}\right)$ & Range \\
\hline Large $(\mathrm{n}=42)$ & $47.16 \pm 38.74$ & $17-243$ \\
Medium \& small $(\mathrm{n}=31)$ & $42.81 \pm 27.92$ & $20-130$ \\
\hline
\end{tabular}

$\mathrm{P}=0.596$ as calculated using the t-test.

Table 6. Comparison of flap type and the mean necrosis area of the divided flaps

\begin{tabular}{|c|c|c|c|c|}
\hline \multirow{2}{*}{ Flap size } & \multicolumn{3}{|c|}{ Partial necrosis of divided flap (№.) } & \multirow{2}{*}{$\begin{array}{l}\text { Area of necrosis (\%), } \\
\quad \text { mean } \pm \mathrm{SD}^{\mathrm{a})}\end{array}$} \\
\hline & Mild (<10\%) & Moderate (11\%-50\%) & Severe (>51\%) & \\
\hline Large $(n=42)$ & 2 & 1 & 1 & $2.38 \pm 9.83$ \\
\hline Medium \& small $(n=31)$ & 2 & 0 & 1 & $2.58 \pm 10.94$ \\
\hline
\end{tabular}

a) $\mathrm{P}=0.935$ as calculated using the t-test. 
provement of appearance, and restoration of function. Notably, to achieve these results, adequate soft tissue coverage must be established, and distant pedicled flaps from the groin and abdomen have been the preferred approach since the 1970s [5]. However, the advent of microsurgery has led to the introduction of fascial free flaps, innervated fasciocutaneous flaps, and arterialized venous flaps as novel options for reconstruction [6-9]. A single bridged free flap is a reconstructive method commonly used in our institution to address the challenges encountered in cases of degloved or amputated stumps of multiple digits. Importantly, it only requires the sacrifice of a single pedicle, thereby generally minimizing operation time. In addition, circulation monitoring for the flap is relatively simple and their harvest results in minimal single-donor site morbidity.

In this study, various donor sites were used based on the size, shape, location, and number of digits of the defect. Nine different types of flaps were used, which were obtained from various regions, ranging from the upper extremities to the lower extremities. Among them, the anterolateral thigh free flap (fasciocutaneous or fascial type) was the most frequently utilized flap (59\% of cases) because it can be used to cover two to five digits of various sizes and was used for all dorsal, volar, and amputation-stump defects. Compared to the other options, it provides adequate gliding of the underlying tendon through stable and healthy soft tissue and can be used in a thin form $[11,12]$. The lateral arm free flap, which was also applied as the fasciocutaneous or the fascial type, was primarily used to reconstruct the dorsal side, along with the venous forearm free flap and hypothenar free flap. Importantly, the thenar free flap was the most common medium-sized flap. A partial second toe pulp free flap was used to treat a pulp defect in two fingertips. In addition to the upper and lower extremities, other areas of the body can be used for hand reconstruction. For example, free tissue options such as the latissimus dorsi can cover large areas, while the serratus flap is effective for smaller defects. Furthermore, the temporal parietal flap is classically used to cover the dorsal side of the hand due to its pliability, dependability, and amenability to tendon excursion [13].

Notably, a single free flap performed over multiple digits requires secondary surgery for flap division. However, in 1933, German et al. [14] found that flaps in dogs had matured sufficiently within 7 days to sustain pedicle division. They examined the blood flow of the flaps and gained insight into the early neovascularization that develops in transplanted tissue. They successfully divided tubed flaps in patients at 10 days. In 1943, Douglas and Buchholz [15] further improved neovascularization of the distal portions of pedicle flaps, finding that flap division was possible at 9-11 days in most instances. In a series of studies, Hynes [16,17] reported success with extremity flaps as early as 9 days. In 1966, Klingenstrom and Nylen [18] successfully transferred tubed skin flaps from the arm to the chest with successful division in only 7 days. Notably, this experience enabled them to routinely divide clinical flaps after intervals of 7-14 days. Another study using two cross-leg flaps found that they were successfully divided after 11 days [9]. A study by Mandelbaum et al. [19] reported successful cross-leg flap division after 4 weeks. Other studies using a pedicled anterolateral thigh and groin flap found that they could be successfully divided at 3-4 weeks $[11,20,21]$. Trovato et al. [22] successfully divided a syndactylizing venous free flap on dorsal digital defects at 3 weeks. Nevertheless, despite evidence that sufficient neovascularization develops earlier than the traditional 3 weeks, many surgeons are content with the success assured at 3 weeks, rather than tempting fate by performing flap division a few days earlier.

We also planned flap division after at least 3 weeks. Flap division was attempted after observing flap capillary refill and any color change at the distal margin. Based on the results of these tests, if revascularization of the flap was considered unclear just before the separation operation, the flap division was delayed. Additionally, in cases of large flaps, such as the anterolateral thigh free flap, most surgeons feel that 3 weeks is too soon to attempt flap division. Based on our experience with different types and sizes of flap divisions, we investigated whether there was a difference in the actual timing of flap division between the two flap size groups. We classified nine types of flaps into a large flap group and a medium and small flap group. In both groups, flap division was successfully achieved after 3 weeks. Although there were cases in which separation surgery was delayed more than 3 weeks, there was no significant difference between the groups. Free flaps were typically used to anastomose one perforator artery, without a significant difference between the two groups. Importantly, we expected that large, thick flaps would have delayed flap division due to insufficient blood flow compared to small flaps. However, this was not the case. These findings suggest that if blood flow to the flap is sufficiently maintained for the time required for revascularization, flap division may be attempted regardless of flap size. In fact, in one case, an anterolateral thigh free flap that was applied over two digits was successfully divided on the 17 th day. Therefore, we believe that division of a large flap is possible within 3 weeks.

In addition, we expected that necrosis at the distal end would be more severe in the large flap group because the distance from the anastomosis site was greater. However, there was no significant difference between the two groups, although severe partial necrosis did develop in two cases (Fig. 3). The first case was a 

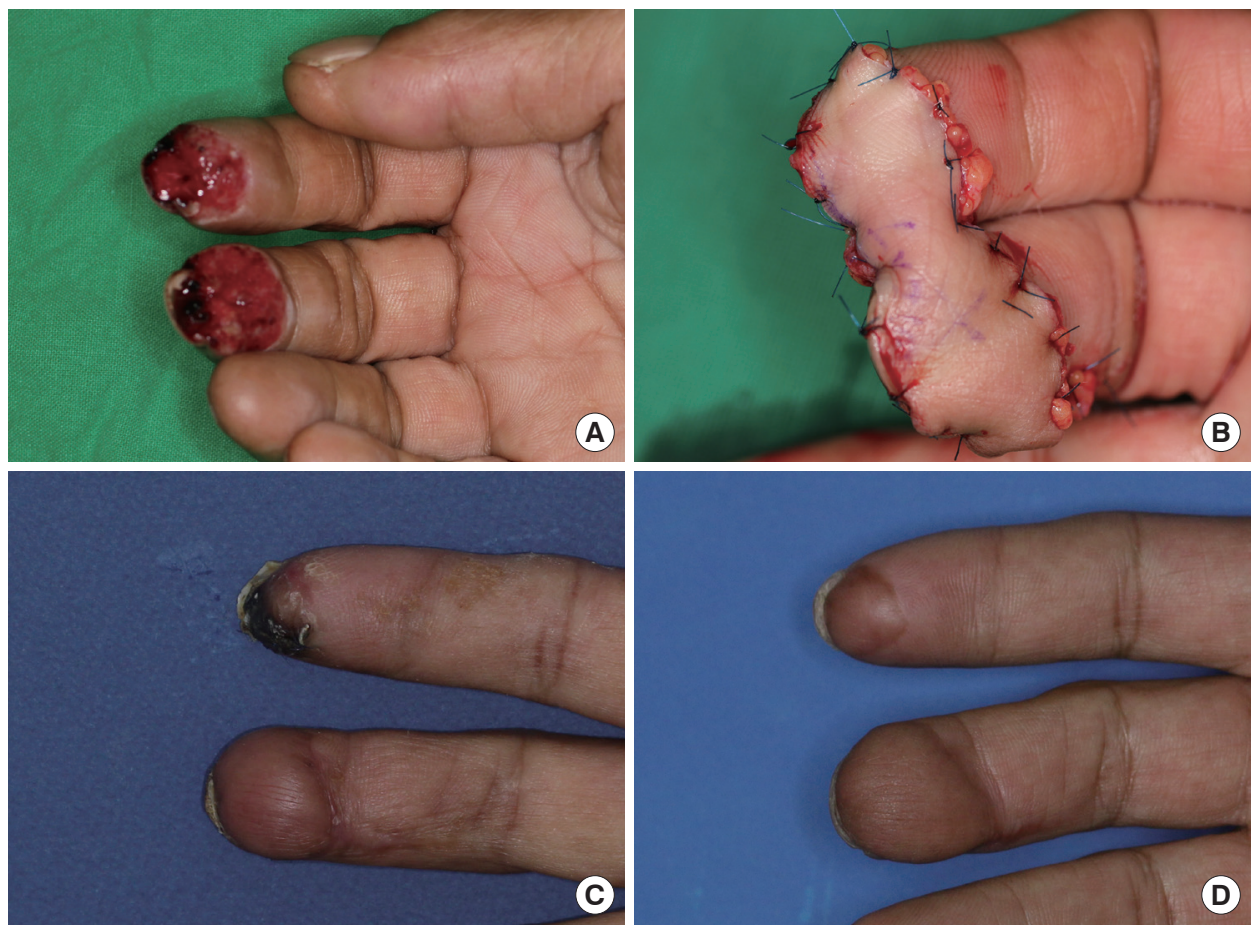

Fig. 3. Case of partial necrosis. (A) A 61-year-old man suffered amputation of his right index and long fingers. A volar oblique pulp defect and bone exposure of the fingers were observed. (B) We harvested a flap with a single pair of perforator artery and vena comitans from the ipsilateral thenar area and anastomosed it to the ulnar digital artery and volar vein of the long finger. (C) Division of the flap was performed 6 weeks after the first surgery. About $60 \%$ of the necrosis that developed was on the ulnar side of the divided flap of the index finger. (D) Three months later, the divided flap had healed without the need for additional surgery.

medium flap division surgery performed at 6 weeks. A thenar free flap was applied on fingertip volar oblique pulp defects across the index and long fingers. The flap was anastomosed to the perforator artery and the vena comitans was anastomosed to the ulnar digital artery and volar vein of the long finger. Division of the flap at the 3rd week was planned, but when clamping in the middle of the flap, the capillary refill time in the distal area was observed to be slow, so surgery was delayed. Division of the flap was performed 6 weeks after the first surgery, but discoloration developed on the ulnar side of the divided flap on the index finger. Eventually, about $60 \%$ necrosis developed, but this was debrided and the flap healed without additional surgery. Importantly, due to the difference in length between the long finger and the index finger, an empty space was created between the bridged flap applied between the two fingertips and the ulnar side of the short index finger. Therefore, the necrosis was thought to be due to a flap design that did not sufficiently adhere to the recipient site. Another case of severe necrosis was similar. An anterolateral thigh free flap was performed on the amputation stump of the fingertip across the index and long fingers. The perforator artery and nerve harvested with the flap were anastomosed to the radial digital artery and nerve of the index finger. One vena comitans anastomosed to the volar vein of the index finger. The flap was anastomosed to a pair of perforator arteries, the vena comitans and nerve to the radial digital artery and the nerve and volar vein of the index finger. For the same reason, division could not be performed at the 3rd week, and was performed at the 34 th week. About $60 \%$ of the divided flap on the radial side of the long finger developed necrosis and was treated with additional skin grafting. This suggests that if the flap and the recipient area are not in sufficient contact, neovascularization will not progress at that site no matter how long it takes, as it likely undergoes scarring or epithelialization. Therefore, to prevent necrosis, one should check whether the flap is in sufficient contact and add additional fixation sutures as needed.

In some patients, the interval of the flap division was prolonged as a result of the surgeon's limited operation schedule, the patient's general condition, or personal reasons. Notably, the majority of patient injuries in this study were caused by industrial accidents. In the case of industrial accidents, patients discharged before the second operation were required to obtain approval from their employer before flap division surgery could be performed. In general, flap division was planned 3 weeks after the free flap operation, but if the flap was considered unstable due to poor circulation, surgery was postponed for a week. The 
aforementioned reasons resulted in a wide distribution in the timing of flap division surgery among the cases.

In summary, our findings demonstrate that delays in flap division were not related to the size of the flap. If the blood flow to the flap has been stable for more than 3 weeks, flap division can be attempted regardless of the flap size.

\section{NOTES}

\section{Conflict of interest}

Jin Soo Kim and Dong Chul Lee are editorial board members of the journal but were not involved in the peer reviewer selection, evaluation, or decision process of this article. No other potential conflicts of interest relevant to this article were reported.

\section{Ethical approval}

The study was approved by the Institutional Review Board of Gwangmyeong Sungae General Hospital (IRB No. KIRB2021-N-002) and performed in accordance with the principles of the Declaration of Helsinki. The informed consent was waived because this study design is a retrospective chart review.

\section{Patient consent}

The patients provided written informed consent for the publication and the use of their images.

\section{Author contribution}

Conceptualization: JS Kim, SY Roh, CH Song, SH Koh, DC Lee, KJ Lee. Data curation: JS Kim, SY Roh, CH Song. Formal analysis: JS Kim, CH Song. Methodology: JS Kim, SY Roh, CH Song, SH Koh, DC Lee. Project administration: JS Kim, SY Roh, CH Song, SH Koh. Writing - original draft: JS Kim, SY Roh, CH Song. Writing - review \& editing: JS Kim, SY Roh, CH Song, SH Koh, DC Lee, KJ Lee.

\section{ORCID}

$\begin{array}{ll}\text { Jin Soo Kim } & \text { https://orcid.org/0000-0003-3369-2974 } \\ \text { Si Young Roh } & \text { https://orcid.org/0000-0002-8625-6124 } \\ \text { Cheon Ho Song } & \text { https://orcid.org/0000-0001-7096-2836 } \\ \text { Sung Hoon Koh } & \text { https://orcid.org/0000-0002-8094-2561 } \\ \text { Dong Chul Lee } & \text { https://orcid.org/0000-0003-4211-6901 } \\ \text { Kyung Jin Lee } & \text { https://orcid.org/0000-0002-9448-8291 }\end{array}$

\section{REFERENCES}

1. Hsu WM, Wei FC, Lin CH, et al. The salvage of a degloved hand skin flap by arteriovenous shunting. Plast Reconstr
Surg 1996;98:146-50.

2. Thomas BP, Katsarma E, Tsai TM. Replantation of total degloving of the hand: case report. J Reconstr Microsurg 2003; 19:217-20.

3. Rodriguez-Lorenzo A, Lin CH, Lin CH, et al. Replantation of a degloved hand with added arteriovenous anastomoses: report of two cases. J Hand Surg Am 2009;34:1864-7.

4. Lo S, Lin YT, Lin CH, et al. A new classification to aid the selection of revascularization techniques in major degloving injuries of the upper limb. Injury 2013;44:331-5.

5. Ulrich D, Pallua N. Treatment of avulsion injury of three fingers with a compound thoracodorsal artery perforator flap including serratus anterior fascia. Microsurgery 2009;29: 556-9.

6. Sukop A, Tvrdek M, Duskova M, et al. Degloving injury: the use of a combination of free fasciocutaneous sensitive flap and pedicle flaps for reconstruction. Acta Chir Plast 2005; 47:107-11.

7. Chen SL, Chou GH, Chen TM, et al. Salvage of completely degloved finger with a posterior interosseous free flap. $\mathrm{Br} \mathrm{J}$ Plast Surg 2001;54:69-71.

8. Takeuchi M, Sakurai H, Sasaki K, et al. Treatment of finger avulsion injuries with innervated arterialized venous flaps. Plast Reconstr Surg 2000;106:881-5.

9. Gatti JE, LaRossa D, Brousseau DA, et al. Assessment of neovascularization and timing of flap division. Plast Reconstr Surg 1984;73:396-402.

10. Kim KS, Kim ES, Hwang JH, et al. Fingertip reconstruction using the hypothenar perforator free flap. J Plast Reconstr Aesthet Surg 2013;66:1263-70.

11. Senda H, Muro H, Terada S, et al. A case of degloving injury of the whole hand reconstructed by a combination of distant flaps comprising an anterolateral thigh flap and a groin flap. J Reconstr Microsurg 2011;27:299-302.

12. Wink JD, Gandhi RA, Ashley B, et al. Flap reconstruction of the hand. Plast Reconstr Surg 2020;145:172e-183e.

13. Brent B, Upton J, Acland RD, et al. Experience with the temporoparietal fascial free flap. Plast Reconstr Surg 1985;76: $177-88$.

14. German W, Finesilver EM, Davis JS. Establishment of circulation in tubed skin flaps: an experimental study. Arch Surg 1933;26:27-40.

15. Douglas B, Buchholz RR. The blood circulation in pedicle flaps: an accurate test for determining its efficiency. Ann Surg 1943;117:692-709.

16. Hynes W. The rapid transfer of abdominal tubed pedicles. Br J Plast Surg 1949;2:202-11.

17. Hynes W. A simple method of estimating blood flow with 
special reference to the circulation in pedicled skin flaps and tubes. Plast Reconstr Surg 1949;4:306.

18. Klingenstrom P, Nylen B. Timing of transfer of tubed pedicles and cross-flaps. Plast Reconstr Surg 1966;37:1-12.

19. Mandelbaum M, Lakhiani C, Lenert JJ. Is 21 days too short? Utility of indocyanine green angiography in predicting successful cross-leg flap division in the compromised lower extremity. Plast Reconstr Surg 2021;147:919e-920e.

20. Zelken JA, Chang NJ, Wei FC, et al. The combined ALT- groin flap for the mutilated and degloved hand. Injury 2015; 46:1591-6.

21. Tuncer S, Findikcioglu K, Ayhan S. Upper extremity reconstruction with pedicled anterolateral thigh perforator flap: a simple modification for a difficult situation.J Plast Reconstr Aesthet Surg 2008;61:1119-21.

22. Trovato MJ, Brooks D, Buntic RF, et al. Simultaneous coverage of two separate dorsal digital defects with a syndactylizing venous free flap. Microsurgery 2008;28:248-51. 